\title{
Letter - ED guideline needs more focus on patient-centered management
}

\author{
Re: CUA guideline: Erectile dysfunction, CUAJ, Oct 2021
}

\section{Geoffrey Hackett'; Mike Kirby, FRCPC'; Jonny Coxon ; ; Asif Muneer, MD, FRCS(Urol); on behalf of the British Society for Sexual Medicine}

'Spire Hospital, Little Aston and Aston University Medical School, Birmingham, United Kingdom; ${ }^{2}$ University of Herffordshire, Centre for Research in Primary \& Community Care \& The Prostate Centre, London, United Kingdom (visiting professor); ${ }^{3}$ ender Identity Clinic, Hammersmith \& The Tavistock \& Portman NHS Foundation Trust, United Kingdom; 4 University College London Hospital, London, United Kingdom

Cite as: Hackett G, Kirby M, Coxon J, et al. Letter - ED guidelines need more focus on patient-centered management. Can Urol Assoc J 2021;15(12):425. http://dx.doi.org/10.5489/cuai.7664

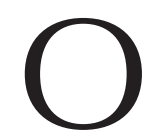
$\mathrm{n}$ behalf of the British Society for Sexual Medicine (BSSM), we must write to say that we find some of the recommendations in the recent Canadian erectile dysfunction (ED) guideline baffling, especially around the issue of daily tadalafil $5 \mathrm{mg}$.

In the introduction, the authors state the importance of quality of life for the patient and the partner but, in the paper, they highlight that they recommend against the use of daily tadalafil $\mathbf{5} \mathbf{~ m g}$. They quote a superiority for daily tadalafil of $0.8 \mathrm{EF}$ points as not clinically significant, yet they only quote comparison studies of 12 weeks or less, whereas studies of six and 12 months show increasing superiority for daily tadalafil, becoming more marked with time. This is especially important in that ED is a long-term condition that usually progresses with age.

We also need to appreciate that clinical trials involved different-sex couples in stable relationships of longer than six months, who received unlimited on-demand medication, whereas in real-life, drugs are prescribed for all sexual orientations, new relationships, and often restricted to one tablet per week.

The International Society for Sexual Medicine (ISSM) syllabus and U.K. National Institute for Health and Care Excellence (NICE) guidance also state that, "Daily tadalafil can be effective in $50 \%$ of men failing on demand medication." Why should men have to tolerate failure that potentially damages their relationship with a partner before receiving a treatment more likely to be effective?
In a later section, on factors that might influence the choice of daily tadalafil, the guideline authors stress that trials show superior spontaneity and sexual confidence, and remove timing concerns, stating that $79.2 \%$ of partners preferred daily treatment, along with a similar proportion of men themselves. Perhaps this tells us that the International Index of Erectile Function (IIEF), developed for clinical trials in restricted groups, is not the most suitable PRO for national prescribing policies. The IIEF does not consider the impact of spontaneous and morning erections on sexuality.

If guidelines are truly "patient-centered," surely the opinion of the couple having sex is the most important patientcentered outcome of all.

The authors also observe that daily tadalafil is a highly effective first-line treatment for lower urinary tract symptoms/ benign prostatic hyperplasia (LUTS/BPH), which is a highly prevalent condition in up to $70 \%$ of men over 55 . By not recommending daily tadalafil, these men will now likely receive an alpha-blocker, which has product warnings of potentially dangerous interactions, especially hypotension with short acting PDE5 inhibitors, not to mention ejaculatory disorders. There are also several longitudinal studies showing reduction in cardiovascular death of $30 \%$, plus improved insulin sensitivity, renoprotection, and even possible reduction in bowel and renal cancer with tadalafil.

Most men in the age groups affected by ED are having sex to give pleasure to themselves and a partner, and the fundamental outcome must be the preference of the couple, not the intellectual satisfaction of their specialist. Surely, this is what patient-centered management is all about. 